\title{
Risk Factors for Colorectal Adenoma - Acknowledging the Burden of NAFLD
}

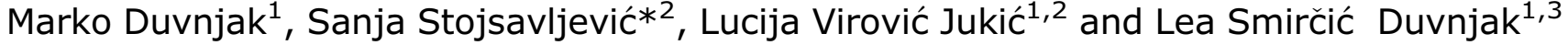 \\ ${ }^{1}$ School of Medicine, University of Zagreb, Zagreb, Croatia; ${ }^{2}$ Department of Gastroenterology and Hepatology, Sisters of Charity \\ University Hospital Center, Zagreb, Croatia; ${ }^{3}$ Department of Endocrinology and Metabolic Diseases, Vuk Vrhovac University Clinic \\ for Diabetes, Merkur University Hospital, Zagreb, Croatia
}

Citation of this article: Duvnjak M, Stojsavljević $S$, Virović Jukić L, Smirčić Duvnjak L. Risk factors for colorectal adenoma Acknowledging the burden of NAFLD. J Clin Transl Hepatol 2019;7(2):97-98. doi: 10.14218/JCTH.2019.00022.

Global trends of diet have changed in Western and Eastern countries, featuring foods that are highly processed, contain saturated fat and carry excessive carbohydrate calories. This, accompanied by lower levels of physical activity, due to a more sedentary lifestyle, and the overweight/obesity epidemic, is the reason we are facing a rise in occurrence of new cases of metabolic syndrome. Furthermore, patients are at substantial risk of developing the well-known complications related to each of the components of metabolic syndrome, those being insulin resistance/diabetes mellitus, arterial hypertension, hyperlipidemia and visceral obesity. ${ }^{1}$

Nonalcoholic fatty liver disease (NAFLD), traditionally regarded as a liver presentation of metabolic syndrome, represents a wide spectrum of liver conditions, ranging from liver steatosis through nonalcoholic steatohepatitis and advanced liver diseases, such as decompensated cirrhosis and hepatocellular carcinoma. NAFLD is also recognized as a risk factor for extrahepatic gastrointestinal (colon, esophagus, stomach, pancreas) and extraintestinal sites of malignancies (kidney in men, breast in women). Patients with NAFLD present with insulin resistance, namely higher levels of circulating insulin effect the insulin/insulin-like growth factor axis that affects cell growth through a potentially carcinogenic pathway, and generally express an adipocytokine profile that promotes a chronic proinflammatory state. ${ }^{2}$ This could represent a link between obesity and promotion of gastrointestinal cancers but more studies are needed to clearly define the entire pathological pathway. ${ }^{3}$

Colorectal cancer (CRC) represents a great health burden worldwide. As such, it is imperative to design an ideal screening plan to detect early CRC and identify precancerous colorectal adenomas (CRAs), in order to reduce the incidence, morbidity, and mortality rates of CRC. The risk factors for CRC include age, family history of CRC, alcohol consumption,

Abbreviations: CAP, controlled attenuation parameter; CRA, colorectal adenoma; CRC, colorectal cancer; NAFLD, nonalcoholic fatty liver disease. Received: 25 June 2019; Accepted: 25 June 2019

*Correspondence to: Sanja Stojsavljević, Department of Gastroenterology and Hepatology, Sisters of Charity University Hospital Center, Vinogradska cesta 29, Zagreb 10000, Croatia. Tel: +385-1-3787-178, Fax: +385-1-3769-067, E-mail: sanja.stojsavljevic1@gmail.com smoking, and increased red meat consumption. ${ }^{4}$ Various screening guidelines for CRC have been proposed by different scientific and clinical societies/organizations, and implemented in the affiliated geographic regions. ${ }^{5-7}$ They all recognize high and low risk groups, mostly depending on the previously mentioned risk factors, but metabolic syndrome and NAFLD, with the aforementioned obesity epidemic have to be taken more seriously in account when creating new guidelines and recommendations. The adoption of future screening programs will be affected by such, but first we must address the lack of adequate noninvasive indicators for CRA.

Li et al. ${ }^{7}$, recently published a retrospective study on 1089 patients (Chinese Hun population), that underwent colonoscopy due to gastrointestinal symptoms (abdominal pain, bowel habit changes, hematochesia, constipation) and iron deficiency anemia. The study participants were divided into groups based on the presence of NAFLD and finding of CRA. The study design addressed the relationship of NAFLD and CRA, recognizing NAFLD as an important risk factor for development of colorectal malignancies. NAFLD was diagnosed in $53.2 \%$ of patients in the CRA group versus $43.8 \%$ in the control (non-NAFLD) group, representing a statistically significant between-group difference $(p=0.008)$. The controlled attenuation parameter (CAP), which indicated liver steatosis measurement obtained with FibroScan, was also significantly higher in the NAFLD group than in the control group ( $p=$ 0.002 ). Interestingly, multifactorial logistic regression analysis showed that sex, NAFLD, CAP, body mass index, triglycerides, serum aspartate aminotransferase, and fasting plasma glucose were the main risk factors tightly associated with the development of CRA. Regarding sex differences, in males, the NAFLD occurrence and CAP values of the CRA group were significantly higher than in the control group ( $p=0.018, p$ $<0.001$ ), which was not observed for the females (all $p>$ 0.05). Independent risk factors of CRA identified for men were NAFLD, CAP, body mass index, serum aspartate aminotransferase, and fasting plasma glucose; for women, the factors were triglycerides and fasting plasma glucose.

While the data of Li et al. ${ }^{8}$ clearly showed the importance of NAFLD as a risk factor for CRA occurrence, there were some limitations to the study itself. The NAFLD diagnosis was not achieved by liver biopsy but via noninvasive methods (ultrasound) and patient selection was limited to patients with gastrointestinal symptoms (excluding general asymptomatic population), which could have led to increased incidence of reported CRA. These limitations are common to 
most studies, but a previous extensive meta-analysis of observational studies that was conducted by Mantovani et al., ${ }^{9}$ involving asymptomatic adults predominantly of Asian descent who underwent screening colonoscopy, suggested that NAFLD is associated with a moderately increased prevalence and incidence of CRA and CRC (random effects odds ratio $=1.4,95 \%$ confidence interval: $1.24-1.57 ; I^{2}=$ $78.8 \%)$, irrespective of the methods used for diagnosing NAFLD (ultrasonography, liver biopsy, proton magnetic resonance spectroscopy). Although the work of Mantovani et al., did not show a clear sex difference for NAFLD-related CRAs, as did the study by Li et al. ${ }^{8}$ the authors suggested that NAFLD may be associated with a higher risk of multiple CRA and more cancers located in the right colon.

Conclusions from the above mentioned studies have potential clinical implication, namely that the diagnosis of NAFLD could identify a subset of individuals who are at higher risk and thus need more careful surveillance; although, more extensive studies are needed to fill the remaining knowledge gaps. Wong et al. ${ }^{10}$ evaluated the higher cost effectiveness of different CRC screening methods (colonoscopy, fecal immunochemical test, flexible sigmoidoscopy) and advocated for a more stringent plan of colonoscopy screening, comprised of screens every 10 years starting at age 50 for patients diagnosed with NAFLD and a positive family history. We expect more epidemiological studies from the East and the West to be carried out, as they could back up the findings for cost effectiveness of more meticulous screening programs for NAFLD patients, which could have significant impacts on clinical and epidemiological outcomes. Nevertheless, the questions have been raised as to whether we are adequately acknowledging the burden of NAFLD in CRA and CRC screening, and whether we are on the right path to obtain appropriate answers.

\section{Conflict of interest}

The authors have no conflict of interests related to this publication.

\section{Author contributions}

Article conception and design (MD, SS), acquisition of data (SS, LVJ), analysis and interpretation of data (MD, SS, LVJ, LSD); drafting of the manuscript (MD, SS), critical revision of the manuscript for important intellectual content (MD, LVJ, LSD), administrative and technical support (MD).

\section{References}

[1] Cornier MA, Dabelea D, Hernandez TL, Lindstrom RC, Steig AJ, Stob NR, et al. The metabolic syndrome. Endocr Rev 2008;29:777-822. doi: 10.1210/er 2008-0024.

[2] Stojsavljević S, Gomerčić Palčić M, Virović Jukić L, Smirčić Duvnjak L, Duvnjak M. Adipokines and proinflammatory cytokines, the key mediators in the pathogenesis of nonalcoholic fatty liver disease. World J Gastroenterol 2014;20:18070-18091. doi: 10.3748/wjg.v20.i48.18070.

[3] Tilg $H$, Moschen AR. Mechanisms behind the link between obesity and gastrointestinal cancers. Best Pract Res Clin Gastroenterol 2014;28:599-610. doi: 10.1016/j.bpg.2014.07.006

[4] Tao S, Hoffmeister M, Brenner H. Development and validation of a scoring system to identify individuals at high risk for advanced colorectal neoplasms who should undergo colonoscopy screening. Clin Gastroenterol Hepatol 2014;12:478-485. doi: 10.1016/j.cgh.2013.08.042.

[5] Sung J], Ng SC, Chan FK, Chiu HM, Kim HS, Matsuda T, et al. An updated Asia Pacific Consensus Recommendations on colorectal cancer screening. Gut 2015;64:121-132. doi: 10.1136/gutjnl-2013-306503.

[6] Provenzale D, Gupta S, Ahnen DJ, Markowitz AJ, Chung DC, Mayer RJ, et al. NCCN Guidelines Insights: Colorectal Cancer Screening, Version 1.2018. Natl Compr Canc Netw 2018;16:939-949. doi: 10.6004/jnccn.2018.0067.

[7] von Karsa L, Patnick J, Segnan N, Atkin W, Halloran S, Lansdorp-Vogelaar I, et al. European guidelines for quality assurance in colorectal cancer screening and diagnosis: overview and introduction to the full supplement publication. Endoscopy 2013;45:51-59. doi: 10.1055/s-0032-1325997.

[8] Li Y, Liu S, Gao Y, Ma H, Zhan S, Yang Y, et al. Association between NAFLD and risk of colorectal adenoma in Chinese Han population. J Clin Transl Hepatol 2019;7:99-105. doi: 10.14218/JCTH.2019.00010.

[9] Mantovani A, Dauriz M, Byrne CD, Lonardo A, Zoppini G, Bonora E, et al. Association between nonalcoholic fatty liver disease and colorectal tumours in asymptomatic adults undergoing screening colonoscopy: a systematic review and meta-analysis. Metabolism 2018;87:1-12. doi: 10.1016/j. metabol.2018.06.004.

[10] Wong MC, Ching JY, Chan VC, Lam TY, Luk AK, Wong SH, et al. Screening strategies for colorectal cancer among patients with nonalcoholic fatty liver disease and family history. Int J Cancer 2016;138:576-583. doi: 10.1002/ijc.29809. 